\title{
On the Transmission-Computation-Energy Tradeoff in Wireless and Fixed Networks
}

\author{
Peter Rost \\ NEC Laboratories Europe \\ Network Divsion, Mobile and Wireless Networks Group \\ 69115 Heidelberg, Germany \\ Email: peter.rost@neclab.eu
}

\author{
Gerhard Fettweis \\ Technische Universität Dresden \\ Vodafone Chair Mobile Communications Systems \\ 01069 Dresden, Germany \\ Email: fettweis@ifn.et.tu-dresden.de
}

\begin{abstract}
In this paper, a framework for the analysis of the transmission-computation-energy tradeoff in wireless and fixed networks is introduced. The analysis of this tradeoff considers both the transmission energy as well as the energy consumed at the receiver to process the received signal. While previous work considers linear decoder complexity, which is only achieved by uncoded transmission, this paper claims that the average processing (or computation) energy per symbol depends exponentially on the information rate of the source message. The introduced framework is parametrized in a way that it reflects properties of fixed and wireless networks alike.

The analysis of this paper shows that exponential complexity and therefore stronger codes are preferable at low data rates while linear complexity and therefore uncoded transmission becomes preferable at high data rates. The more the computation energy is emphasized (such as in fixed networks), the less hops are optimal and the lower is the benefit of multi-hopping. On the other hand, the higher the information rate of the singlehop network, the higher the benefits of multi-hopping. Both conclusions are underlined by analytical results.
\end{abstract}

Index Terms-Computation energy, transmission energy, computation-transmission-energy tradeoff, multi-hop networks

\section{INTRODUCTION}

\section{A. Energy-Efficiency in Multi-Hop Networks}

Recently WWRF Chair M.A. Uusitalo announced his vision of the future wireless world 1 . One of his major technological visions is that until the year 20177 trillion wireless devices will be used by 7 billion users. Mobile communication engineers face a multitude of challenges to integrate this tremendous number of nodes such as more demanding requirements on the radio resource management, packet routing, and energy efficiency. The latter is in the focus of this paper, which analyzes the interplay of the energy consumption of the transceiver path (transmission energy) and the data processing unit (computation energy). We introduce a framework and draw conclusions, which can be equally applied to fixed networks, cellular networks, and low-complexity sensor networks.

There exists a comprehensive literature analyzing the transmission energy consumption in a wireless network such as the seminal work in [1], where a bursty protocol has been introduced. A bursty protocol shortens the online time of a node, concentrates the transmitted energy on a shorter

\footnotetext{
${ }^{1}$ Presentation is available on http://wireless-world-research.org/
}

time interval, and increases the signal-to-noise ratio (SNR) in analog multi-hop networks. Intuitively, a bursty transmission reduces the constant energy as the online-time of the node is reduced. On the other hand, the information rate is increased, which implies that the data processing unit potentially requires more energy, if the required average computation power per symbol scales super-linearly. This inherent tradeoff is not only of importance for energy-limited terminals, which have only a limited energy budget, but it is of equal importance for fixed networks where data aggregation and bursty transmission are valid alternatives to reduce the energy consumption. Due to the tremendous number of sensor nodes and the requirement for a power source such as a battery, even small energy savings per node imply a significant 'green potential'. This inherent tradeoff of transmission and computation energy is in the focus of this work and we explore how an appropriate choice of the packet length and number of nodes in a network can reduce the overall energy consumption.

\section{B. Related Work}

In previous work such as [2], [3] the computation energy of a network has been only considered under the assumption of a linear dependence of rate and energy. In [2], the authors investigate the lifetime of a network where individual nodes collect and deliver data. In particular, it considers the transmission energy, the source behavior, network size, and also how much computation energy is required to receive a bit, which relates linearly to the information rate. Similarly, [3] also analyzed the network lifetime and applied a linear model for the computation energy. As we discuss later, a linear model does not suitably reflect the case of coded transmission, since we rather face an exponential dependency. The routing problem in wireless networks with per-bit processing-power has been analyzed in [4], where again the processing energy depends linearly on the information rate.

\section{Contribution and Outline}

This work introduces a framework to analyze and to assess the tradeoff of computation and transmission energy in multihop networks such as relay-based cellular networks, sensor networks, but also fixed networks with intermediate gateways and routers. We discuss the inter-play of both and show how 


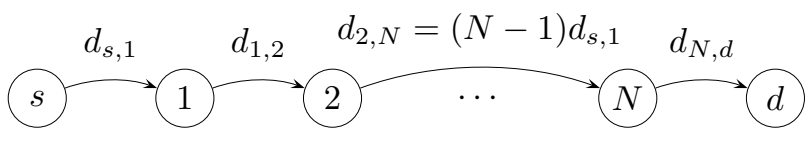

Fig. 1. System setup with $N+2$ nodes distributed at equal distances.

packet length, data rate, network size, and the functional description of the computation complexity affects this relationship and depict potentials for the optimization of a network's energy consumption. The underlying system model will be introduced in Section [II. Based on this model we derive the normalized computation and transmission energy of a decodeand-forward (also called store-and-forward) based multi-hop network in Section III. The tradeoff of both is illustrated in Section IV and the paper is concluded in Section $\mathrm{V}$.

\section{System MODEL}

Before we present the transmission-computation-energy tradeoff in Section III, we successively introduce our channel model, resource model, and energy model.

\section{A. Channel Model}

We consider a network composed of the source node $s=$ 0 , the set of $N$ intermediate nodes $\mathcal{R}=[1 ; N]$, and the destination node $d=N+1$ as illustrated in Fig. 1. This paper focuses on an additive white Gaussian noise (AWGN) channel with fixed channel gain. The channel input at node $n$ is the complex Gaussian random process $\mathrm{X}_{n} \sim \mathcal{C N}\left(0, P_{t x, n}\right)$ with average per-symbol power $P_{t x, n}$. Let the distance between two nodes $n, n^{\prime} \in[0 ; N+1]$ be $d_{n, n^{\prime}}$. Then the channel gain between both nodes is $h_{n, n^{\prime}}=d_{n, n^{\prime}}^{-\alpha / 2}$ with path loss exponent $\alpha$. For the sake of notational simplification we assume in this paper that all nodes are distributed at equal distance between $s$ and $d$ such that

$$
d_{n, n^{\prime}}=\frac{\left|n^{\prime}-n\right|}{N+1} d_{s, d}
$$

This assumption is rarely fulfilled in wireless sensor networks, but is of particular relevance in fixed networks. In addition, the conclusions drawn in this paper do not depend on this assumption but rather on the non-linear nature of path loss as well as the different complexity. In the AWGN model, the channel output at node $n^{\prime}$ is given by

$$
\mathrm{Y}_{n^{\prime}}=\sum_{n \in[0 ; N+1] \backslash n^{\prime}} h_{n^{\prime}, n} \mathrm{X}_{n}+\mathrm{Z}_{n^{\prime}} \text {, }
$$

where $\mathrm{Z}_{n^{\prime}} \sim \mathcal{C} \mathcal{N}\left(0, \sigma^{2}\right)$ is the AWGN with $\sigma^{2}=1$ throughout this paper.

We consider in this work a network of full-duplex terminals in order to introduce the computation-transmission-energy tradeoff. Full-duplex is easily implemented in fixed networks where both links are physically separated using different physical cables. However, wireless applications imply a halfduplex constraint on the deployed nodes and therefore an inherent rate-loss, which renders multi-hop transmission less beneficial.

\section{B. Resource Model and Means of Normalization}

Our analysis compares the multi-hop setup with a singlehop reference system $(N=0)$ with source-destination distance $d_{\text {ref }}=d_{s, d}=1$, and the reference transmission power $P_{t x, s}=P_{t x, \text { ref. }}$. Let us assume that the source node has a fixed amount of data collected, which is mapped to the codeword $W_{\text {ref }}$ with overall $\left\|W_{\text {ref }}\right\|$ bits net-data and must be delivered to the destination. Assume that $T_{\text {ref }}<\infty$ exclusive resource elements (available channel uses) are assigned to the source node, which can correspond to time slots in an TDMA system or exclusive bandwidth in an FDMA system. Without loss of generality we refer in the following to $T_{\text {ref }}$ as the number of symbols in time. In order to reliably communicate $W_{\text {ref }}$, the source must transmit with an average information rate per symbol $R_{\text {ref }}=\left\|W_{\text {ref }}\right\| / T_{\text {ref }}$, i.e., with each channel access on average $R_{\text {ref }}$ bits must be transmitted. In the previously described Gaussian AWGN channel model the average information rate per symbol is described by $R_{\text {ref }}=\mathrm{C}\left(P_{t x \text {,ref }} / \sigma^{2}\right)$ with $\mathrm{C}(x)=\log _{2}(1+x)$. Throughout this paper, we use the number of bits $\left\|W_{\text {ref }}\right\|$ as means of normalization and require that each protocol must reliably communicate $W_{\text {ref }}$ using at most $T_{\text {ref }}$ time symbols.

This normalization offers the degree of freedom to adjust the number of used time symbols $T^{\prime}<T_{\text {ref }}$, which implies that a node uses only parts of the assigned resources. However, in order to deliver the same amount of data, the rate must be increased such that $R^{\prime}=\left(T_{\text {ref }} / T^{\prime}\right) R_{\text {ref }}$ as per channel access a higher number of information bits must be communicated. In the following part, we introduce our energy model and how the overall transmission and computation energy depend on the active time period $T^{\prime}$.

\section{Energy Model and Bursty Transmission}

From the previous introduction we can immediately state that the transmission energy in the reference system is given by $E_{t x \text {,ref }}=P_{t x \text {,ref }} \cdot T_{\text {ref }}$. Under the assumption that the destination decodes the transmission also in a time interval $T_{\text {ref }}$ (in order to avoid an accumulation of packets), it must also decode the data with rate $R_{\text {ref }}$. Motivated by convolutional codes, which can be decoded using a trellis representation [5] of the encoder's state space, we claim that the decoding complexity for each time symbol is exponential in the information rate $R_{\text {ref }}$. This behavior is caused by the fact that also the state space and the number of possible state transitions per channel access in the decoder-trellis expands exponentially with the product of constraint length and $R_{\text {ref }}$. Previous work only considered linear complexity, which implies an uncoded transmission and an actual performance loss that can be expressed by a constant SNR gap as introduced in [6, pp. 66]. We apply an SNR gap between exponential and linear complexity of $5 \mathrm{~dB}$ [6], [7], which implies that a system with linear decoding complexity must invest $5 \mathrm{~dB}$ higher transmission power in order to achieve the same performance.

The computation power can be expressed by $P_{c \text {, ref }} \sim$ $c_{1} c_{3}^{c_{2} R_{\text {ref }}}$ where the constants $c_{j}$ are decoder specific. For the sake of simplicity, we consider in our work $c_{1}=c_{2}=1$ 
(we neglect the constraint length as it remains the same for all $T^{\prime}$ ) and $c_{3}=2$ such that the computation energy for a packet of length $T_{\text {ref }}$ is given by $E_{c \text {,ref }}=T_{\text {ref }} \cdot 2^{R_{\text {ref }}}$, which is used as reference value for our evaluation of multihop networks. The actual parametrization changes for different coding schemes and inherently affects the quantitative results of the transmission-computation-energy tradeoff although the qualitative conclusions are not affected.

Assume the transmission length used by node $n$ is $T^{\prime}<$ $T_{\text {ref }}$, then the transmission energy is scaled such that

$$
T_{\mathrm{ref}} \mathrm{C}\left(\frac{P_{t x, n}}{\sigma^{2}}\right)=T^{\prime} \mathrm{C}\left(\frac{P_{t x, n}^{\prime}}{\sigma^{2}}\right)
$$

is fulfilled. This implies that the bursty protocol with $T^{\prime}<T_{\text {ref }}$ requires the transmission power

$$
P_{t x, n}^{\prime}\left(\delta_{t}=T^{\prime} / T_{\text {ref }}\right)=\sigma^{2}\left(\left(1+\frac{P_{t x, n}}{\sigma^{2}}\right)^{1 / \delta_{t}}-1\right)
$$

in order to satisfy the constraint that the same amount of data must be communicated. In addition, also the computation energy increases as the rate $R^{\prime}=\mathrm{C}\left(\frac{P_{t x, n}^{\prime}}{\sigma^{2}}\right)$ implies an exponential scaling of the computation energy. Let $\Delta_{r}=$ $R^{\prime}-R_{\text {ref }}$, then the computation energy is given as

$$
E_{c, n}^{\prime}=2^{\Delta_{r}} E_{c, n}
$$

which deviates from algorithms with linear complexity where $E_{c, n}^{\prime}$ scales linearly with the ratio of $R^{\prime}$ and $R_{\text {ref }}$. In the following, all derivations are presented for exponential complexity while the corresponding equations for linear complexity can be easily obtained using a linear model in (5).

\section{Normalized ENERgy in Multi-Hop Networks}

In order to capture the tradeoff between transmission and computation energy in multi-hop networks, we use the decodeand-forward protocol introduced in [8] with node-cooperation and non-coherent transmission. Given the power assignment vector $\left[P_{t x, 0}, P_{t x, 1}, \ldots P_{t x, N}\right]$ the maximum achievable endto-end rate is given by [8]

$$
R \leq \min _{1 \leq n \leq N+1} \mathrm{C}\left(\frac{1}{\sigma^{2}} \sum_{k=0}^{n-1} h_{n, k}^{2} P_{t x, k}\right) .
$$

In the following, we define the normalized transmission and computation energy of a multi-hop network compared to a single-hop transmission for a fixed number of resource elements. On this basis, we extend the framework to define the normalized energy for a flexible and optimized number of resource elements in our multi-hop network.

\section{A. Normalized Energy for Fixed $T_{\text {ref }}$}

The rate on the first hop is given by

$$
R_{0}=\mathrm{C}\left(\frac{P_{t x, 0}}{\sigma^{2}}\right)
$$

with the source power given as a function of the reference power:

$$
P_{t x, 0}=(N+1)^{-\alpha} P_{\text {ref }} .
$$

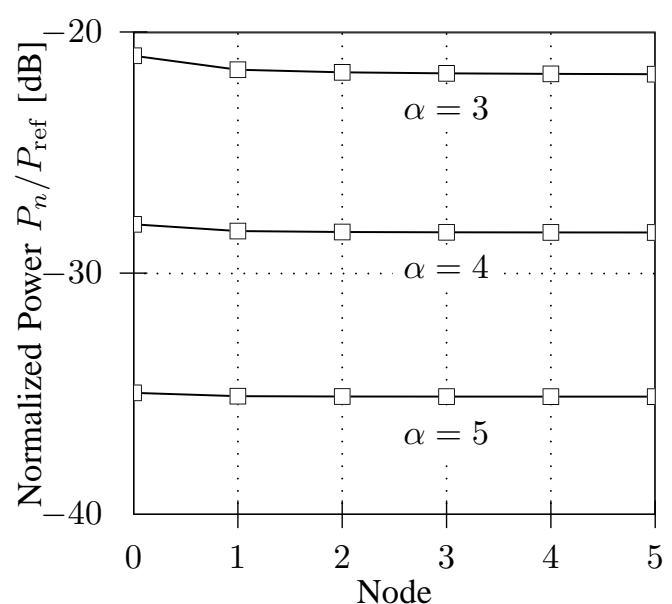

Fig. 2. Power assignment example for 5 nodes and different path loss values

In order to achieve the same rate on the second hop, the transmission power of the second terminal must be chosen such that

$$
\begin{aligned}
h_{0,1}^{2} P_{t x, 0} & =h_{0,1}^{2} P_{t x, 1}+h_{0,2}^{2} P_{t x, 0} \text { with } h_{n, n^{\prime}}=d_{n, n^{\prime}}^{-\alpha / 2} \\
P_{t x, 1} & =\left(1-2^{-\alpha}\right) P_{t x, 0} .
\end{aligned}
$$

This can be generalized for the transmission power of node $n$ as follows

$$
\begin{aligned}
h_{0,1}^{2} P_{t x, 0} & =\sum_{k=0}^{n} h_{n+1, k}^{2} P_{t x, k} \\
& =\sum_{k=0}^{n}(n+1-k)^{-\alpha} h_{0,1}^{2} P_{t x, k} \\
P_{t x, n} & =P_{t x, 0}-\sum_{k=0}^{n-1}(n+1-k)^{-\alpha} P_{t x, k}
\end{aligned}
$$

$P_{t x, n}$ is strictly monotonically decreasing in $n$ as illustrated in Fig. 2. Hence, the power assignment $P_{t x, n}=P_{t x, 0}$ provides an achievable but suboptimal solution for wireless networks (in case of $\alpha=4$ the maximum difference in Fig. 2 is about $0.35 \mathrm{~dB}$ per node) and provides the exact solution for fixed networks where no cooperation gain can be exploited. The normalized network-wide transmission energy for packet length $T_{\text {ref }}$ is

$$
\begin{aligned}
E_{\text {tx }, \text { norm }}\left(\delta_{t}=1\right) & =\frac{T_{\text {ref }} \sum_{k=0}^{N} P_{t x, k}}{T_{\text {ref }} P_{\text {ref }}} \leq \frac{(N+1) P_{t x, 0}}{P_{\text {ref }}} \\
& =(N+1)^{1-\alpha}
\end{aligned}
$$

and the normalized computation energy is given by

$$
E_{\mathrm{c}, \text { norm }}\left(\delta_{t}=1\right)=\frac{T_{\text {ref }} \sum_{n=1}^{N+1} 2^{R_{\text {ref }}}}{T_{\text {ref }} 2^{R_{\text {ref }}}}=N+1,
$$

which already shows that the computation energy grows faster in $N$ than the transmission energy and therefore eventually becomes the dominant term for large $N$. 


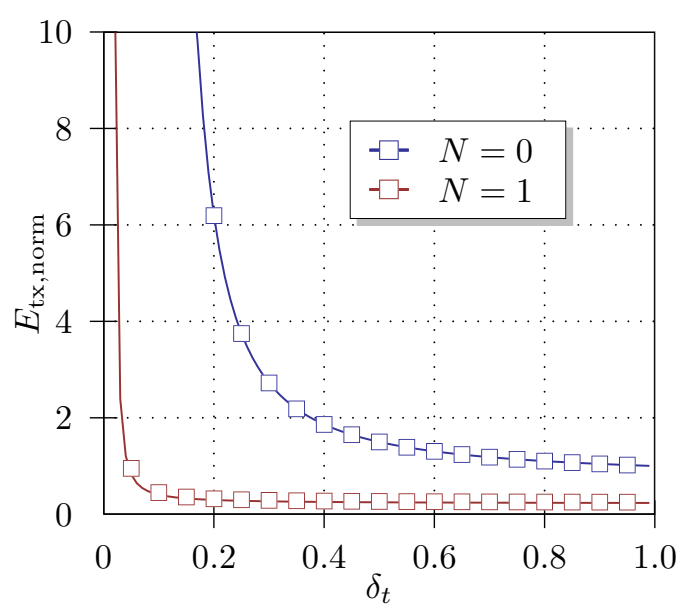

Fig. 3. Normalized transmission energy for different relative $\delta_{t}$ and number of relays $N$. Markers indicate the approximation derived in 19] and lines give the exact solution as derived in [18. This example uses $\alpha=3, P_{\text {ref }}=1$, $\sigma^{2}=1$, which implies $R_{\text {ref }}=1$.

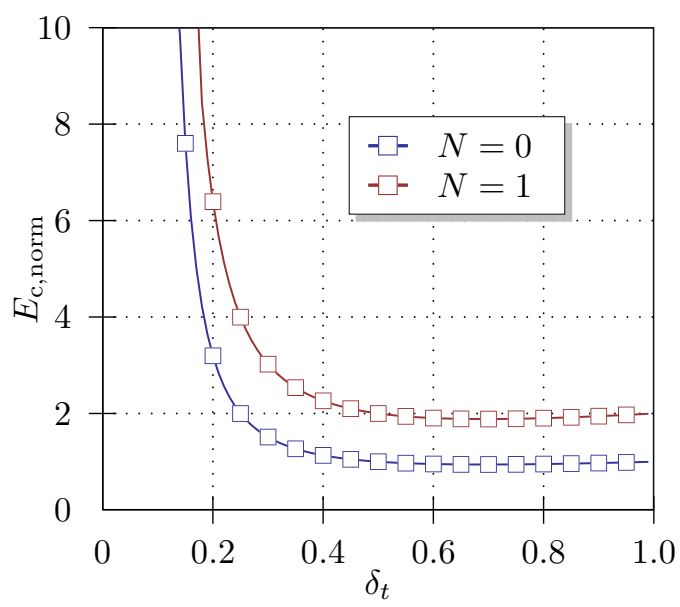

Fig. 4. Normalized transmission energy for different relative packet lengths using the expression in 22 and for reference rate $R_{\text {ref }}=1$.

\section{B. Bursty Transmission using $T^{\prime}<T_{\mathrm{ref}}$}

If the packet length used by the multi-hop protocol is chosen as $T^{\prime}<T_{\text {ref }}$ for each hop, then the normalized transmission energy is according to (4) and with $\delta_{t}=T^{\prime} / T_{\text {ref }}$ given by

$$
\begin{aligned}
E_{\mathrm{tx}, \mathrm{norm}}\left(\delta_{t}\right) & =\delta_{t} \frac{\sum_{n=0}^{N} P_{t x, n}^{\prime}\left(\delta_{t}\right)}{P_{\mathrm{tx}, \mathrm{ref}}} \\
& =\delta_{t} \frac{\sum_{n=0}^{N} \sigma^{2}\left(\left(1+\frac{P_{t x, n}}{\sigma^{2}}\right)^{1 / \delta_{t}}-1\right)}{P_{\mathrm{tx}, \mathrm{ref}}} \\
& \leq \delta_{t}(N+1)^{1-\alpha} \frac{\sigma^{2}\left(\left(1+\frac{P_{t x, 0}}{\sigma^{2}}\right)^{1 / \delta_{t}}-1\right)}{P_{t x, 0}} .
\end{aligned}
$$

An example for $E_{\mathrm{tx}, \text { norm }}\left(\delta_{t}\right)$ is shown in Fig. 3 for $\alpha=3$, $P_{t x, \text { ref }}=1$, and $\sigma^{2}=1$, which gives $R_{\text {ref }}=1$.
Similarly, the computation energy is given by

$$
\begin{aligned}
E_{\mathrm{c}, \text { norm }}\left(\delta_{t}\right) & =\delta_{t} \frac{\sum_{n=1}^{N+1} 2^{R_{\text {ref }}} 2^{\Delta_{r}}}{2^{R_{\text {ref }}}} \\
& =\delta_{t}(N+1) 2^{\Delta_{r}}=\delta_{t} 2^{\Delta_{r}} E_{\mathrm{c}, \text { norm }}(1) .
\end{aligned}
$$

The rate difference $\Delta_{r}=R^{\prime}-R_{\text {ref }}$ must be such that $R_{\text {ref }} T_{\text {ref }}=R^{\prime} T^{\prime}$, which implies

$$
E_{\mathrm{c}, \text { norm }}\left(\delta_{t}\right)=\delta_{t} 2^{R_{\mathrm{ref}}\left(1 / \delta_{t}-1\right)} E_{\mathrm{c}, \text { norm }}(1) .
$$

An example for the normalized computation energy is shown in Fig. 4 for the reference rate $R_{\text {ref }}=1$. Interestingly, assume $T_{\text {ref }}=2 T^{\prime}\left(\delta_{t}=0.5\right)$ and $R_{\text {ref }}<1$ then a bursty transmission will not consume more computation energy than the direct transmission. On the other hand, if the rate increases to $R_{\text {ref }}>1$, the bursty transmission will increase the required computation energy.

\section{Optimal Packet Length $T^{\prime}$}

We can easily identify the packet length, which minimizes the computation energy in the multi-hop network (once we chose $N$ ) to be

$$
\begin{aligned}
T_{\mathrm{c}, \text { opt }}^{\prime} & =\arg \min _{0<T^{\prime} \leq T} E_{\mathrm{c}, \text { norm }}\left(T^{\prime} / T_{\text {ref }}\right) \\
\frac{\mathrm{d} E_{\mathrm{c}, \text { norm }}\left(T^{\prime} / T_{\text {ref }}\right)}{\mathrm{d} T^{\prime}} & \stackrel{!}{=} 0 \\
T_{\mathrm{c}, \text { opt }}^{\prime} & =T_{\text {ref }} \min \left((\ln 2) R_{\text {ref }}, 1\right) .
\end{aligned}
$$

We know from (19) that the transmission energy can only increase for $T^{\prime}<T_{\text {ref }}$ such that the optimal $T^{\prime}$, which minimizes the overall energy consumption, must be in the interval $\left[T_{\mathrm{c}, \mathrm{opt}}^{\prime} ; T_{\mathrm{ref}}\right]$ and depends on the ratio of $E_{\mathrm{tx} \text {,norm }}$ and $E_{\mathrm{c}, \text { norm }}$. However, $T_{\mathrm{c}, \mathrm{opt}}^{\prime}$ provides a good lower bound on the optimal packet length and only depends on the reference rate $R_{\text {ref, }}$, which simplifies its computation.

The overall energy required by the network is given as

$$
\begin{aligned}
E_{\mathrm{sum}}\left(R_{\mathrm{ref}}\right)= & E_{\mathrm{c}, \text { norm }}\left(R_{\mathrm{ref}}\right) \cdot E_{\mathrm{c}, \mathrm{ref}}\left(R_{\mathrm{ref}}\right) \\
& +E_{\mathrm{tx}, \text { norm }}\left(R_{\mathrm{ref}}\right) \cdot E_{\mathrm{tx}, \mathrm{ref}}\left(R_{\mathrm{ref}}\right) .
\end{aligned}
$$

Now let $E_{\mathrm{c}, \text { ref }}=\eta_{\mathrm{ref}}\left(R_{\mathrm{ref}}\right) E_{\mathrm{tx}, \mathrm{ref}}$, which relates the computation and transmission energy for a single-hop system depending on the actual rate. Using (4) and (5), the function $\eta_{\text {ref }}\left(R_{\text {ref }}\right)$ can be expressed depending on a system-specific reference value $\eta_{\text {ref }}(1)$ (as shown in the appendix). The overall consumed energy in the multi-hop network is now given as

$$
\begin{aligned}
E_{\mathrm{sum}}\left(R_{\mathrm{ref}}\right)= & E_{\mathrm{c}, \text { norm }} \cdot \eta_{\mathrm{ref}}\left(R_{\mathrm{ref}}\right) E_{\mathrm{tx}, \mathrm{ref}} \\
& +E_{\mathrm{tx}, \text { norm }} \cdot E_{\mathrm{tx}, \mathrm{ref}},
\end{aligned}
$$

where $E_{\mathrm{c}, \text { norm }}$ and $E_{\mathrm{tx}, \text { ref }}$ are the normalized energies for reference rate $R_{\text {ref }}$ (which are omitted here to avoid any confusion with $\delta_{t}$ ). The normalized sum-energy can be expressed by

$$
E_{\mathrm{sum}, \text { norm }}\left(R_{\mathrm{ref}}\right)=\frac{E_{\mathrm{c}, \mathrm{norm}} \cdot \eta_{\mathrm{ref}}\left(R_{\mathrm{ref}}\right)+E_{\mathrm{tx}, \mathrm{norm}}}{\left.1+\eta_{\mathrm{ref}}\left(R_{\mathrm{ref}}\right)\right)} .
$$




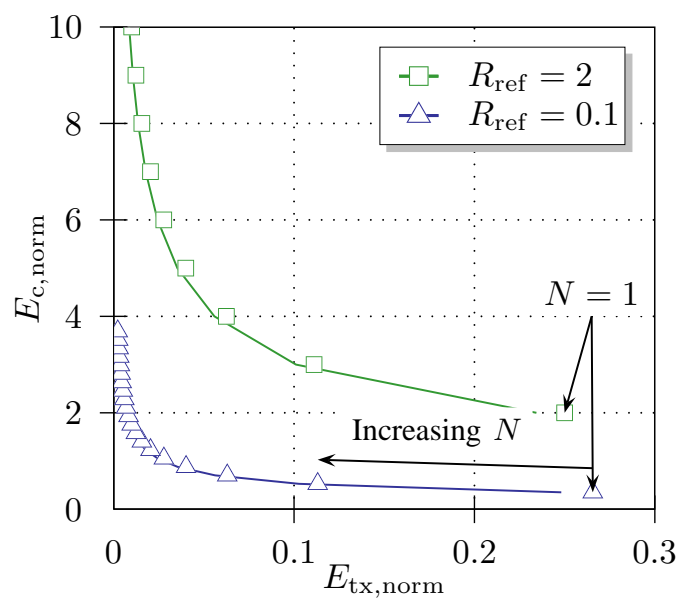

Fig. 5. Tradeoff of normalized computation and transmission energy for $N \in[1 ; 30]$ relays and reference rates $R_{\text {ref }}$. Lines show the exact solution for wireless networks and markers show the exact solution for fixed networks (which also serves as lower bound for wireless networks). Furthermore, $\alpha=3$ and $\sigma^{2}=1$.

\section{RESULTS}

Based on the previously described framework, we discuss in this section results for the transmission-computation-energy tradeoff. We focus thereby on two particular aspects. Firstly, we analyze the jointly achievable transmission-computationenergy curve under a given throughput-constraint, and secondly, we discuss the optimal network size and energy savings potential depending on the reference rate $R_{\text {ref }}$.

- Strange point at 1.5 value of Rref

\section{A. Transmission-Computation-Energy Tradeoff}

Fig. 5 shows the achievable transmission-computationenergy curve for low-rate transmission $\left(R_{\text {ref }}=0.1\right)$ and highrate transmission $\left(R_{\text {ref }}=2\right)$. Lines indicate the exact solution for wireless networks using $T_{\text {c,opt }}^{\prime}$ in 18 while markers show the solution for fixed networks derived in (19), which also serves as approximated solution for wireless networks. Each marker indicates one particular setup with $N$ relay nodes where higher $N$ result in lower normalized transmissionenergy and higher normalized computation-energy.

The minimum normalized computation-energy is lower for the low-rate transmission than for the high-rate transmission, which indicates that in multi-hop networks with fixed $N$ the relative computation-energy savings are higher for low rates. Consider the low-rate transmission and the slope of the curve. In the case of linear complexity, the computational energy doubles with every additional hop irrespective of the packet length. Hence, the normalized computational energy for a linearly complex algorithm has slope 1 in $N$ and is significantly higher than for exponential complexity. On the other hand, for high-rate transmission the computational energy for exponential complexity also increases linearly in $N$ as $T_{\text {c,opt }}^{\prime}=T_{\text {ref. }}$. Therefore, low-rate transmission implies that coding with higher complexity is preferable over a less complex codes, while for high-rate transmission as in fixed

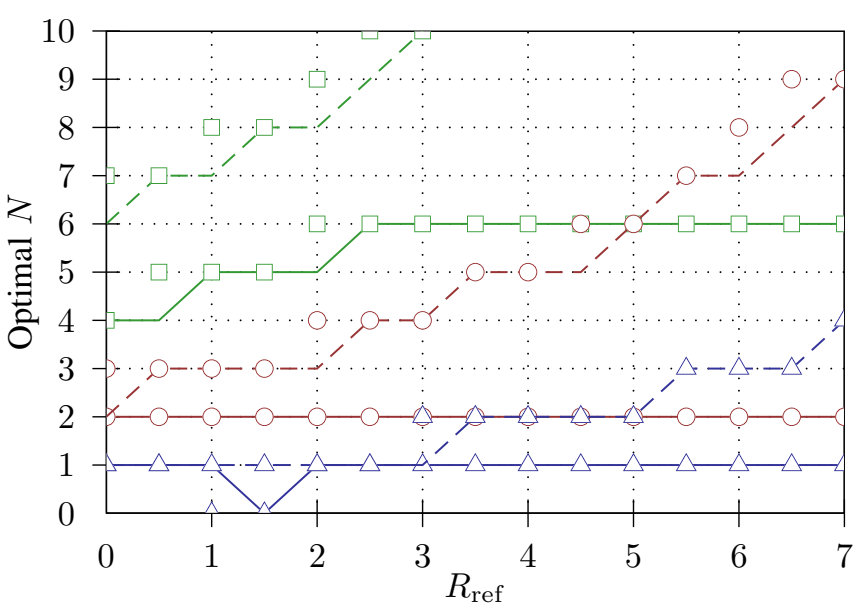

(a) Network size

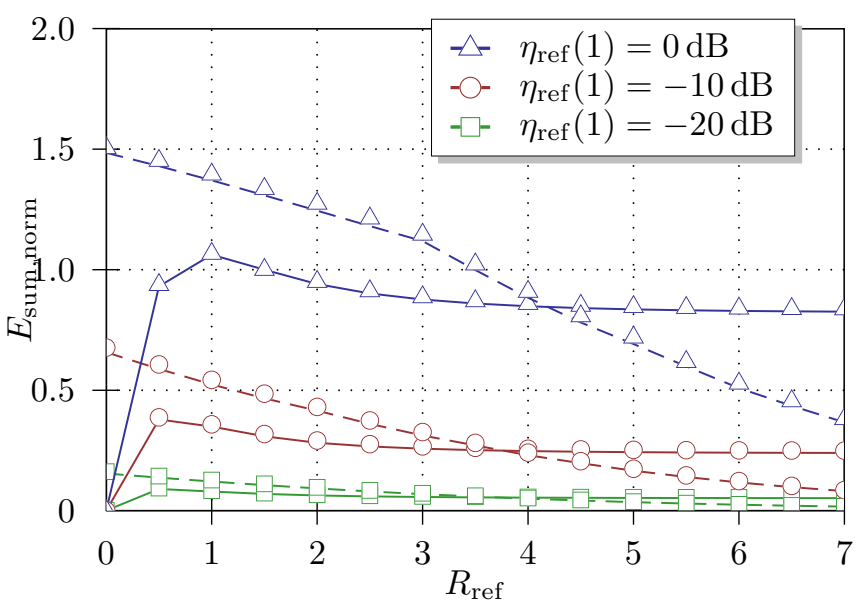

(b) Sum Energy normalized

Fig. 6. Minimum normalized sum-energy and the optimal number of relay nodes depending on the reference rate $R_{\text {ref }}$. Markers show the solution for fixed networks and lines show the solution for wireless networks. Dashed lines give the results for linear complexity while solid lines give the results for exponential complexity. Again, $\alpha=3, \sigma^{2}=1$.

networks less complex codes are preferable with respect to the transmission-computation-energy tradeoff.

\section{B. Optimization of the Overall Energy}

Fig. 6 shows the normalized sum-energy according to 28 for three different values of $\eta_{\text {ref }}(1)$ and the optimal number of relay nodes, which minimizes the sum-energy for a particular value of $\eta_{\text {ref }}(1)$ and $R_{\text {ref }}$ and $T_{c, o p t}$ given in (25). In Fig. 6 solid lines indicate the results for exponential complexity and dashed lines for linear complexity relative to the same common reference power (after application of a $5 \mathrm{~dB}$ SNR gap). Markers again show the solution for fixed networks while lines show the solution for wireless networks.

Fig. 6-a) shows the optimal $N$ depending on $\eta_{\text {ref }}(1)$ and $R_{\text {ref }}$. The higher the computation energy compared to the transmission energy (reflected by a higher $\eta_{\text {ref }}(1)$ ) the lower the optimal $N$. With an increasing emphasis on the computation energy, it becomes the dominant part of the sum-energy, which renders a higher number of nodes less 
beneficial. In addition, if the reference rate is increasing, the optimal number of nodes is also increasing in order to counteract the exponentially increasing transmission energy. The slope of this increase is higher for $\eta_{\text {ref }}(1)=-20 \mathrm{~dB}$ than for $\eta_{\text {ref }}(1)=0 \mathrm{~dB}$. The latter refers to fixed networks where computation energy contributes more significantly to the sum-energy than in wireless networks. In addition, networks with linear computational complexity prefer more hops than networks with exponential complexity. Further consider the optimal $N$ at $R_{\text {ref }}=1.5$ for $\eta_{\text {ref }}(1)=0 \mathrm{~dB}$. It reaches an minimum at this point as the normalized sum-energy is greater than 1 and therefore relaying is not optimal for this case. However, this is different for linear complexity as we apply a $5 \mathrm{~dB}$ shift, which then renders relaying beneficial again.

Fig. 6(b) shows the minimum normalized sum-energy using $T_{c, \text { opt }}^{\prime}$ in (25) and using the optimal $N$ depicted in Fig. 6a). The lowest value of $E_{\text {sum,norm }}$ is obtained for $\eta_{\text {ref }}(1)=$ $-20 \mathrm{~dB}$ as the transmission energy can be significantly reduced and the computation energy does not become a dominating part with increasing $N$. With increasing reference rates, the transmission energy in the single-hop network becomes a more dominant part of the sum-energy. Due to the significant transmission power savings in multi-hop networks, also the normalized sum-energy declines with increasing $R_{\text {ref }}$. This implies that multi-hop transmission is more useful in scenarios with high data rates and less complex decoders and encoders. We can further see that at low rates the sum-energy is higher for linear complexity than for exponential complexity while at higher rates linear complexity is again preferable with respect to the sum-energy. By contrast, for $\eta_{\text {ref }}(1)=-20 \mathrm{~dB}$ (wireless case) both linear and exponential complexity achieve similar sum-energy performance (as the transmission power is dominating).

\section{Conclusions and Future Challenges}

This paper introduced and analyzed the tradeoff of the energy required for decoding and processing transmissions and the energy necessary to transmit a message. We derived a framework, which showed that for increasing emphasis on the computation energy (increasing $\eta_{\text {ref }}(1)$ ), multi-hop protocols are less beneficial to reduce the network-wide spent energy, while for increasing emphasis on the transmission energy (increasing reference rate $R_{\text {ref }}$ ) they become more beneficial. The comparison of linear and exponential complexity showed that more complex encoding is preferable at low data rates while low complex encoding is preferable at high rates with respect to the transmission-computation-energy tradeoff. In addition, using different weighting of the transmission and computation energy as for instance in wireless and fixed networks, we showed that a smaller number of hops in fixed networks is preferable due to the significant computation energy while in wireless networks more hops are preferable due to the dominating transmission energy.

Among the next challenges is the question for the optimal complexity-function rather than for the optimal protocol or number of hops. If a functional expression of the SNR-gap depending on the computational complexity can be found, the optimal computation complexity for both wireless and fixed networks can be determined.

\section{APPENDIX}

In section $\amalg I I-C$ we introduced the function $\eta_{\text {ref }}\left(R_{\text {ref }}\right)$, which relates the computation and transmission energy in the single-hop reference system such that $E_{\mathrm{c}, \text { ref }}=$ $\eta_{\text {ref }}\left(R_{\text {ref }}\right) E_{\text {tx }, \text { ref }}$. Assume that for reference rate $R_{\text {ref }}=1$ the function is predefined as a system-specific parameter. At this rate the source must transmit on the direct link with power $P_{\mathrm{tx}, \mathrm{ref}}(1)$. Assume that the rate is now given by $R^{\prime}=$ $\delta_{r} R_{\text {ref }}$, then the transmission energy is given by $E_{\mathrm{tx}, \mathrm{ref}}\left(1 / \delta_{r}\right)$, where we applied (4). The computation energy is given by $2^{\Delta_{r}} E_{\mathrm{c}, \mathrm{ref}}(1)$ as given by (5). Hence, we can derive $\eta_{\mathrm{ref}}\left(R_{\mathrm{ref}}\right)$ as

$$
\begin{aligned}
2^{\Delta_{r}} E_{\mathrm{c}, \mathrm{ref}}(1) & =\eta_{\mathrm{ref}}\left(R_{\mathrm{ref}}\right) \cdot E_{\mathrm{tx}, \mathrm{ref}}\left(1 / \delta_{r}\right) \\
2^{\Delta_{r}} \eta_{\mathrm{ref}}(1) E_{\mathrm{tx}, \mathrm{ref}}(1) & =\eta_{\mathrm{ref}}\left(R_{\mathrm{ref}}\right) \cdot E_{\mathrm{tx}, \mathrm{ref}}\left(1 / \delta_{r}\right) \\
\eta_{\mathrm{ref}}\left(R_{\mathrm{ref}}\right) & =\eta_{\mathrm{ref}}(1) \cdot \frac{2^{\Delta_{r}} E_{\mathrm{tx}, \mathrm{ref}}(1)}{E_{\mathrm{tx}, \mathrm{ref}}\left(1 / \delta_{r}\right)} .
\end{aligned}
$$

\section{REFERENCES}

[1] A. Avestimehr and D. Tse, "Outage capacity of the fading relay channel in the low-SNR regime," IEEE Transactions on Information Theory, vol. 53, no. 4, pp. 1401-1415, April 2007.

[2] M. Bhardwaj and A. Chandrakasan, "Upper bounds on the lifetime of sensor networks," in IEEE International Conference on Communications, Helsinki, Finland, June 2001.

[3] E. Duarte-Melo and M. Liu, "Analysis of energy consumption and lifetime of heterogeneous wireless sensor networks," in IEEE Global Communications Conference, Taipei, Taiwan, November 2002.

[4] W. Heinzelman, A. Chandrakasan, and H. Balakrishnan, "Energy-efficient communication protocol for wireless microsensor networks," in 33rd Hawaii International Conference on System Sciences, Hawaii, USA, January 2000.

[5] V. Tarokh, N. Seshadri, and A. Calderbank, "Space-time codes for high data rate wireless communications: Performance criterion and code construction," IEEE Transactions on Information Theory, vol. 44, no. 2 , pp. 744-765, March 1998.

[6] B. Li and G. Stüber, Orthogonal Frequency Division Multiplexing for Wireless Communications. Birkhäuser, 2006.

[7] W. Yu, D. Varodayan, and J. Cioffi, "Trellis and convolutional precoding for transmitter-based interference presubtraction," IEEE Transactions on Information Theory, vol. 53, no. 7, pp. 1220-1230, July 2005.

[8] L.-L. Xie and P. Kumar, "A network information theory for wireless communication: Scaling laws and optimal operation," IEEE Transactions on Information Theory, vol. 50, no. 5, pp. 748-767, May 2004. 